\title{
SOME EXTREMAL PROBLEMS FOR VECTOR BUNDLES
}

\author{
V. V. MAKEEV
}

\begin{abstract}
Analogs of well-known problems and theorems on convex bodies are considered for the case where the convex bodies are replaced by continuous fields of convex bodies in vector bundles.
\end{abstract}

\section{$\S 1$. INTRODUCTION}

By a convex body $K \subset \mathbb{R}^{n}$ we mean a compact convex set with nonempty interior. In what follows, $V(K)$ denotes the volume of $K \subset \mathbb{R}^{n}$ and $S(K)$ the area of $K \subset \mathbb{R}^{2}$.

We say that a field of convex bodies (f.c.b.) or a field of figures (f.c.f.) is given in a vector bundle $\gamma: E \rightarrow B$ if in each fiber of the bundle we have a convex body (or a figure in the case of two-dimensional fibers) that continuously depends on the fiber.

The present paper is a continuation of the paper [1], where various geometric problems on continuous fields of convex bodies in vector bundles were studied.

A typical extremal problem on convex bodies (see [2]) is to find the maximal value of a certain $G$-invariant functional $f$ on the class of convex bodies $K \subset \mathbb{R}^{n}$, where $G$ is a subgroup of the group $\operatorname{Aff}\left(\mathbb{R}^{n}\right)$ of affine transformations of $\mathbb{R}^{n}$.

Let $f$ be a $G$-invariant functional on the class of convex bodies $K \subset \mathbb{R}^{n}$, and let $\gamma: E \rightarrow B$ be an $n$-dimensional vector bundle with structure group $G$. Let $s$ be an f.c.b. in the bundle $\gamma$, and let $s(b), b \in B$, be a convex body belonging to the field in question and lying in the fiber $\gamma^{-1}(b)$. In this paper, we consider the problem of finding $\inf _{s} \sup _{b} f(s(b))$ for various functionals $f$. In the survey [2], many functionals $f$ of geometric origin defined on convex bodies in $\mathbb{R}^{n}$ were considered. In the present paper, we only deal with some of them.

We consider f.c.b. in the most important tautological bundle

$$
\gamma_{k}^{n}: E_{k}\left(\mathbb{R}^{n}\right) \rightarrow G_{k}\left(\mathbb{R}^{n}\right),
$$

where $G_{k}\left(\mathbb{R}^{n}\right)$ is the Grassmann manifold of $k$-dimensional planes passing through $O \in$ $\mathbb{R}^{n}$. In this bundle, the fiber over a $k$-plane $g \in G_{k}\left(\mathbb{R}^{n}\right)$ is the same plane regarded as a $k$-dimensional subspace of $\mathbb{R}^{n}$.

The most important examples of f.c.b. of geometric nature in $\gamma_{k}^{n}$ are the fields of cross sections of a convex body $K \subset \mathbb{R}^{n}$ that contains the point $O$ by planes in $G_{k}\left(\mathbb{R}^{n}\right)$ (or projections of $K$ to the planes in $G_{k}\left(\mathbb{R}^{n}\right)$ ). Therefore, every theorem on f.c.b. in $\gamma_{k}^{n}$ implies a similar theorem on $k$-dimensional cross sections or projections of a convex body $K \subset \mathbb{R}^{n}$.

Below, we focus on the simplest case of f.c.b. in $\gamma_{2}^{n}$, and especially on the bundle $\gamma_{2}^{3}$, which is the first nontrivial case.

2000 Mathematics Subject Classification. Primary 52A99, 51M20.

Key words and phrases. Field of convex bodies, mass distribution, normed plane. 


\section{$\S 2$. UNIVERSALLY INSCRIBED POLYGONS}

Besicovitch [6] proved that every plane convex figure $K$ admits an inscribed affine image of a regular hexagon. Later, various proofs of this fact were given by other mathematicians (see [2]).

In [1], it was conjectured that each f.c.b. in the bundle $\gamma_{2}^{n}$ contains a figure that has an inscribed (circumscribed) affine-regular $(2 n+2)$-gon and also a figure that has an inscribed (circumscribed) regular $2 n$-gon.

We note that, for $n=2$, this conjecture gives the Besicovitch theorem mentioned above, together with the well-known theorem stating that every convex plane figure admits an inscribed square, and also the corresponding dual theorems. We also note that the famous Schnirel'man theorem [7] says that one can inscribe a square in every regular Jordan plane curve.

Asymptotically, the conjecture stated above was actually proved in [8], which implies that, for each prime $p<2 n-2$, every f.c.b. in the bundle $\gamma_{2}^{n}$ contains a figure that has an inscribed (circumscribed) regular $p$-gon. For further refinements of this estimate, see [9].

However, only in one case has the conjecture been proved completely. In [10], it was shown that every f.c.f. in $\gamma_{2}^{3}$ contains a figure circumscribed about (inscribed in) an affine-regular octagon.

Thus, the first open case of the conjecture states that, for $n=3$, there is a convex figure admitting an inscribed (circumscribed) regular hexagon.

It has been noticed by N. Yu. Netsvetaev that this case of the conjecture implies the Besicovitch theorem.

Indeed, for a given convex figure $K \subset \mathbb{R}^{2}$, consider a cylinder $C \subset \mathbb{R}^{3}$ with base $K$ and with height considerably greater than the diameter of the base. In the bundle $\gamma_{2}^{3}$, we consider the f.c.f. formed by the cross sections of $C$ by planes passing through an interior point $O$ of $C$ equidistant from the bases of $C$. Observe that the figure cut out by a plane touching a base of the cylinder does not admit any inscribed (circumscribed) regular hexagon, because such a figure is very stretched by the construction of $C$. Let $S$ be a regular hexagon inscribed in (circumscribed about) a cross section that does not touch the bases of $C$, and let $\pi: C \rightarrow K$ be the orthogonal projection onto a base $K$ of $C$. Then $\pi(S)$ is an affine-regular hexagon inscribed in (circumscribed about) $K$.

Remark. In $\S 9$, a slight modification of the above argument will allow us to prove that if every f.c.f. in $\gamma_{2}^{n+1}$ contains a figure that can be inscribed in (circumscribed about) a regular $(2 n+2)$-gon, then every f.c.f. in $\gamma_{2}^{n}$ contains a figure that can be inscribed in (circumscribed about) an affine-regular $(2 n+2)$-gon.

It is not known whether every Jordan plane curve admits an inscribed square. However, the following statement is valid.

Theorem 1. Every continuous field of Jordan curves in the bundle $\gamma_{2}^{3}$ contains a curve circumscribed about a square.

Proof. Let $s$ be a continuous section of $\gamma_{2}^{3}$ such that, for every plane $x \in G_{2}\left(\mathbb{R}^{3}\right)$, the point $s(x)$ is inside the Jordan curve lying in the fiber $x$. We show that some curve of the field is circumscribed about a square with center at the corresponding point $s(x)$ of its fiber.

We prove this for smooth fields of smooth Jordan curves and a smooth section $s$. We prove that the number of such squares is odd in the typical situation.

Consider the bundle $\gamma: E \rightarrow G_{2}\left(\mathbb{R}^{3}\right)$ associated with $\gamma_{2}^{3}$, in which the fiber over a plane $g \in G_{2}\left(\mathbb{R}^{3}\right)$ is the four-dimensional manifold of squares in this plane. For a typical 
field of smooth Jordan curves in $\gamma_{2}^{3}$, the squares inscribed in the curves of this field form a smooth compact two-dimensional manifold $C$. Let

$$
\pi: E \rightarrow E_{2}\left(\mathbb{R}^{3}\right)
$$

be the projection mapping a square to its center. Then $\pi(C)$ realizes a two-dimensional cycle in $E_{2}\left(\mathbb{R}^{3}\right)$. This cycle intersects a typical fiber in $E_{2}\left(\mathbb{R}^{3}\right)$ at an odd number of points, because an odd number of squares is inscribed in a typical smooth Jordan plane (see [7]). Thus, $\pi(C)$, as well as $s\left(G_{2}\left(\mathbb{R}^{3}\right)\right)$, realize the generator of the group $H_{2}\left(E_{2}\left(\mathbb{R}^{3}\right) ; \mathbb{Z}_{2}\right) \cong \mathbb{Z}_{2}$, and their intersection index is 1 modulo 2, which implies the claim.

The general case is obtained from the above by passing to the limit with the help of approximating the continuous field of Jordan curves and the section $s$ by smooth fields of smooth Jordan curves and smooth sections, respectively. The theorem is proved.

\section{§3. Ellipticity measures ANd symmetries of CONVEX Bodies}

3.1. Ellipticity measures. It is well known (see [2]) that, for every convex body $K \subset$ $\mathbb{R}^{n}$, there exists an ellipsoid $E$ such that $E \subset K \subset n E$, where $n E$ is the ellipsoid homothetic to $E$ and having the same center; the coefficient of homothety is $n$. Here, as an outer ellipsoid $E_{1}$ we can take the (unique) Levner ellipsoid that has minimal volume and contains the body $K$; in this case, we have $\frac{1}{n} E_{1} \subset K$. As an inner ellipsoid $E_{2}$ we can take the (unique) ellipsoid that has maximal volume and lies in $K$; then $K \subset n E$. Under our assumptions, $f_{1}(K)=V(K) / V\left(E_{1}\right)$ and $f_{2}(K)=V\left(E_{2}\right) / V(K)$ are affine-invariant ellipticity measures of the convex body $K \subset \mathbb{R}^{n}$, which (by [2]) means that they are continuous, take values in [0,1], and are equal to 1 if and only if $K$ is an ellipsoid.

Theorem 2. Every f.c.f. in the bundle $\gamma_{2}^{3}$ contains a figure $K_{1}$ with $f_{1}\left(K_{1}\right) \geq 2 / \pi$ such that in its fiber there is an ellipse $E_{1}$ with

$$
\frac{E_{1}}{\sqrt{2}} \subset K_{1} \subset E_{1}
$$

and also a figure $K_{2}$ with $f_{2}\left(K_{2}\right) \geq \pi / 4$ such that in its fiber there is an ellipse $E_{2}$ with

$$
E_{2} \subset K_{2} \subset \sqrt{2} E_{2} \text {. }
$$

All these estimates are sharp.

Proof. It is easily seen that for the role of $K_{1}$ we can take a figure that belongs to the given field and is inscribed in an affine-regular octagon $\Omega_{1}$ (see [10]), and as $E_{1}$ we can take an ellipse circumscribed about $\Omega_{1}$. It is easy to check that $E_{1} / \sqrt{2} \subset K_{1} \subset E_{1}$ and

$$
\sqrt{2} S\left(K_{1}\right) \geq S\left(\Omega_{1}\right)=\frac{2 \sqrt{2}}{\pi} S\left(E_{1}\right) .
$$

For the role of $K_{2}$ we can take a figure that belongs to the given field and admits an inscribed affine-regular octagon $\Omega_{2}$ (see [10]), and as $E_{2}$ we can take an ellipse inscribed in $\Omega_{2}$. It is easy to check that $E_{2} \subset K_{2} \subset \sqrt{2} E_{2}$ and

$$
\frac{8(\sqrt{2}-1)}{\pi} S\left(E_{2}\right)=S\left(\Omega_{2}\right) \geq 2(\sqrt{2}-1) S\left(K_{2}\right) .
$$

To prove that the above estimates are sharp, consider an f.c.f. in $\gamma_{2}^{3}$ consisting of the cross sections of a tetrahedron by planes passing through its interior point. Sharpness follows immediately from the fact that those cross sections are either triangles or quadrangles. 
Remarks. 1. Some sharp estimates for f.c.f. in the bundles $\gamma_{2}^{n}$ for $n$ close to primes can be found in $[1,3,9]$.

2. The following important problem remains unsolved: is it true that, for every $a<1$ and every $k \geq 3$, there is $N$ such that for all $n \geq N$ an arbitrary f.c.b. in the bundle $\gamma_{k}^{n}$ contains a body $K$ with $f_{i}(K) \geq a, i=1,2$ ? Clearly, the positive answer is a nonintegrable version of the well-known Dvoretzky theorem [11]. For $k=2$ the answer is in the positive [3].

3.2. Minkowski symmetry measure. The well-known Minkowski-Radon theorem says that every convex body $K \subset \mathbb{R}^{n}$ contains an interior point $O$ such that the ratio of the distances from $O$ to each pair of parallel support planes for $K$ is at least $1: n$ (see [2]). It is also known that this is equivalent to the fact that every convex body $K \subset \mathbb{R}^{n}$ contains an interior point $O$ such that every chord of $K$ passing through $O$ is divided by $O$ in the ratio of at least $1: n$ (see [2]).

Theorem 3. Every f.c.f. in the bundle $\gamma_{2}^{3}$ contains a figure $K$ with interior point $O$ such that each chord of $K$ passing through $O$ is divided by $O$ in the ratio of at least $1: \sqrt{2}$. Moreover, the ratio of the distances from $O$ to every pair of parallel support planes for $K$ is at least $1: \sqrt{2}$.

Proof. As $K$, we can take any figure in the f.c.f. in question that has an inscribed (or circumscribed) affine-regular octagon, and the role of $O$ can be played by the center of this octagon.

The author does not know any sharp estimate in this theorem.

Remarks. 1. In [1], it was proved that, for every $a<1$ and a positive integer $k$, there exists $N$ such that, for $n \geq N$, each f.c.b. in $\gamma_{k}^{n}$ contain a body $K$ with an interior point $O$ such that each chord of $K$ passing through $O$ is divided by $O$ in the ratio of at least $a: 1$.

2. The results of [9] imply that, for $n \geq 4$, every f.c.f. in $\gamma_{2}^{n}$ contains a figure $K$ that has an interior point $O$ such that each chord of $K$ passing through $O$ is divided by $O$ in the ratio of at least $\cos 2 \pi / p: 1$, where $p$ is the greatest prime less than $2 n+2$.

Both remarks are valid also for the ratios of the distances from $O$ to each pair of parallel support planes for $K$.

3.3. Kovner, Bezikovich, and Estermann symmetry measures. The well-known Kovner-Bezikovich theorem states that every convex figure $K \subset \mathbb{R}^{2}$ contains a centrallysymmetric figure with area at least $\frac{2}{3} S(K)$ (see [2]).

It is well known that every convex figure $K \subset \mathbb{R}^{2}$ lies in a centrally-symmetric convex figure of area not exceeding $2 S(K)$. (Apparently, the first proof of this fact was obtained by Estermann in [2].)

Theorem 4. In every f.c.f. in the bundle $\gamma_{2}^{3}$, there is a figure $K_{1}$ containing a centrallysymmetric convex figure with area at least $(2 \sqrt{2}-2) S\left(K_{1}\right)$; also, there is a figure $K_{2}$ lying in a centrally-symmetric figure of area not exceeding $\sqrt{2} S\left(K_{2}\right)$.

Proof. Indeed, in every f.c.f. in $\gamma_{2}^{3}$ there is a figure $K_{1}$ in which an affine-regular octagon with area at least $(2 \sqrt{2}-2) S\left(K_{1}\right)$ is inscribed (see Subsection 3.1).

Similarly, in every f.c.f. in $\gamma_{2}^{3}$ there is a figure $K_{2}$ about which an affine-regular octagon with area not exceeding $\sqrt{2} S\left(K_{2}\right)$ is circumscribed (see Subsection 3.1).

Remark. 1. The estimates given in Theorem 4 are certainly not sharp.

2. Theorem 6 of [1] shows that, for every positive $a<1$ and a positive integer $k$, there is $N$ such that, for every positive integer $n>N$, each f.c.b. in the bundle $\gamma_{k}^{n}$ contains a 
body $K$ that contains a centrally-symmetric convex body of volume not exceeding $V(K)$ and lies in a body of volume not exceeding $V(K) / a$.

3 . Towards the proof of the conjecture discussed in $\S 2$, one can use the results of $[1,3,8,9]$ to obtain good estimates for approximations of figures belonging to an f.c.f. in $\gamma_{2}^{n}$ by centrally symmetric convex figures.

4. In [12], it was proved that every convex body $K \subset \mathbb{R}^{3}$ contains an affine image of a circular cylinder with volume at least $\frac{4 \pi}{27 \sqrt{3}} V(K)$. In [13], it was proved that every convex body $K \subset \mathbb{R}^{3}$ lies in an affine image of a regular octagonal pyramid with volume at most $3 \sqrt{2} V(K)$. Both estimates cannot be refined if $K$ is a tetrahedron.

\section{$\S 4 . k$-PARTITE POINTS OF A CONTINUOUSLY DISTRIBUTED MASS AND THE NEUMANN-RADO THEOREM}

4.1. $k$-partite points. It is well known that any continuously distributed mass on a plane can be split into four parts of equal mass by a pair of perpendicular lines.

R. Buck and E. Buck [14] proved that every measurable figure $K \subset \mathbb{R}^{2}$ has a 6 -partite point, i.e., a point for which there exist three lines that pass through this point and subdivide $K$ into 6 parts of equal area. Every continuously distributed finite mass in a plane has a 6 -partite point [2].

We say that a point $A \in \mathbb{R}^{2}$ is $2 k$-partite for a finite continuously distributed mass $m$ if there exist $k$ lines that pass through $A$ and split $m$ into $2 k$ equal parts. We say that a mass distribution field (m.d.f.) is given in a vector bundle $\gamma: E \rightarrow B$ if in each fiber we have a continuously distributed finite mass that depends continuously on the fiber.

Theorem 5. For every m.d.f. in $\gamma_{2}^{3}$, there is a fiber for which the corresponding mass has an 8-partite point.

Proof. In [15], it was proved that every continuously distributed mass in $\mathbb{R}^{3}$ can be orthogonally projected to a plane so as to possess an 8-partite point. The proof extends to the case of an arbitrary m.d.f. in $\gamma_{2}^{3}$.

Remark. The results of [15] imply that, for each m.d.f. in $\gamma_{2}^{n}$, there is a fiber with a $2(n+1)$-partite point for even $n$ and a fiber with a $2 n$-partite point for odd $n \geq 5$. Apparently, for odd $n$ there is also a fiber with a $2(n+1)$-partite point.

In $[1, \S 6]$ it was conjectured that, for every m.d.f. in the bundle $\gamma_{2}^{n}$, there is a fiber $g \subset$ $E_{2}\left(\mathbb{R}^{n}\right)$ with a regular $2 n$-gon (affine-regular $2(n+1)$-gon) such that the rays connecting the center of the polygon with its vertices split the mass distributed in the plane $g$ into equal parts. For $n=2$, we obtain precisely the statements mentioned at the beginning of this section. If $n>2$, then only an "affine" version of the conjecture for $n=3$ is proved, which is a refinement of the theorem on the existence of an 8-partite point for an m.d.f. in the bundle $\gamma_{2}^{3}$. The first unproved case here states that, for every m.d.f. in $\gamma_{2}^{3}$, there is a fiber such that the mass distributed in it can be split into six equal parts by three lines passing through a common point and forming angles of $\pi / 3$.

In the same way as in $\S 2$, it can be proved easily that the "affine" part of the conjecture follows from another conjecture, saying that the existence of the required affine-regular $2(n+1)$-gon for an m.d.f. in $\gamma_{2}^{n}$ follows from the existence of a regular $2(n+1)$-gon for an m.d.f. in $\gamma_{2}^{n+1}$.

4.2. Neumann-Rado theorem. The well-known Neumann-Rado theorem states that for every finite mass uniformly distributed in $\mathbb{R}^{n}$, there is a point such that each hyperplane passing through it splits the mass in the ratio of at least $1: n$. 
Theorem 6. For every m.d.f. in $\gamma_{2}^{3}$, there exists a fiber $g \subset E_{2}\left(\mathbb{R}^{3}\right)$ and a point $A$ in it such that every line in $g$ passing through $A$ splits the mass distributed in $g$ in the ratio of at least $3: 5$.

Proof. Any 8-partite point $A$, existing in a fiber of $\gamma_{2}^{3}$ by Theorem 5, has the required property. Indeed, each half-plane bounded by a line that belongs to this fiber and passes through $A$ contains three of the eight sectors into which four lines passing through $A$ divide the plane of the fiber. Each sector contains one eighth of the mass, and therefore, the half-plane contains at least $3 / 8$ of the mass in the fiber.

Remarks. 1. The author does not know any sharp estimate in Theorem 6 .

2. From the above remark to Theorem 5 , it follows that, for $n>3$ and an arbitrary m.d.f. in the bundle $\gamma_{2}^{n}$, there is a plane $g \subset E_{2}\left(\mathbb{R}^{n}\right)$ and a point $A \in g$ such that every line passing through $A$ splits the mass distributed in $g$ in the ratio of at least $(n-1):(n+1)$ for odd $n$ and in the ratio of at least $n:(n+2)$ for even $n$.

3. The author has no answer to the following fundamental question: is it true that, for every $a<1$ and a positive integer $k$, there exists $N$ with the following property: for every positive integer $n \geq N$ and an arbitrary m.d.f. in the bundle $\gamma_{k}^{n}$, there exists a fiber $g \subset E_{k}\left(\mathbb{R}^{n}\right)$ and a point $A \in g$ such that every line in $g$ passing through $A$ splits the mass distributed in the fiber in the ratio of at least $a: 1$.

\section{$\S 5$. The Length of The Unit CIRCLE IN A NORMED PlANE}

It is well known (see $[16,17])$ that the length of a circle of radius 1 in a normed plane is at least 6 and at most 8 .

The author does not know whether for every Finsler metric in the bundle $\gamma_{2}^{3}$, i.e., in the case where in each fiber we have a norm continuously varying with the fiber, there exists a fiber in which the unit circle has length $2 \pi$. Nevertheless, the following statement is valid.

Theorem 7. For every Finsler metric in $\gamma_{2}^{3}$, there is a fiber with unit circle of length at least $20 \sin \pi / 10$, and also a fiber with unit circle of length at most $20 \sin \pi / 10 \cdot \sec \pi / 5$.

Proof. In [18], it was proved that every continuous field of centrally-symmetric convex figures in the bundle $\gamma_{2}^{3}$ contains a figure circumscribed about an affine-regular decagon and also a figure inscribed in an affine-regular decagon. Simple considerations show that the first figure has perimeter not less than $20 \sin \pi / 10$ and the perimeter of the second figure does not exceed $20 \sin \pi / 10 \cdot \sec \pi / 5$.

Remark. The results of [9] imply that, for every Finsler metric in the bundle $\gamma_{2}^{n}$, there is a fiber with unit circle of length at least $4 p \sin \frac{\pi}{2 p}$, and also a fiber with unit circle of length at most $4 p \sin \frac{\pi}{2 p} \cdot \sec \pi / p$, where $p$ is the greatest prime less than $2 n$.

Diskant and Shcherba $[28,29]$ considered the problem concerning the perimeter of a nonsymmetric unit circle in a plane.

Let $K$ be a convex figure for which the origin $O \in \mathbb{R}^{2}$ is an interior point.

The Minkowski distance or gauge function for the pair $(K, O)$ is the function

$$
g(\bar{x})=\inf \{t>0 \mid \bar{x} \in t K\} .
$$

Thus, the figure $K$ plays the role of a "unit circle" with "center" $O$.

By the length of a broken line $A_{1} \ldots A_{n}$ we mean

$$
\ell\left(A_{1} \ldots A_{n}\right)=\sum_{i=1}^{n-1} g\left(\overline{A_{i} A_{i+1}}\right) .
$$




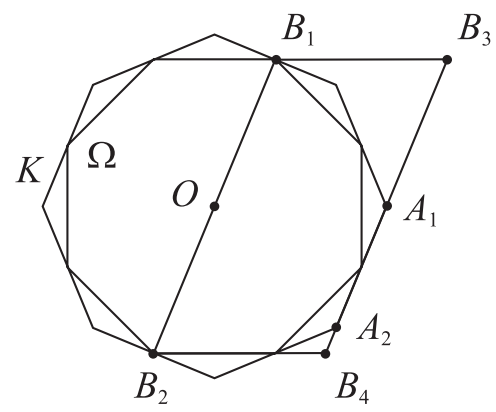

Figure 1

Observe that, in general, the numbers $\ell\left(A_{1} \ldots A_{n}\right)$ and $\ell\left(A_{n} \ldots A_{1}\right)$ are distinct; they always coincide only in the case where $O$ is the center of symmetry of $K$.

In the case where $K$ is a convex polygon, we denote by $p_{+}(K, O)$ and $p_{-}(K, O)$ the length of its boundary passed counterclockwise and clockwise, respectively. By continuity, we can define $p_{+}(K, O)$ and $p_{-}(K, O)$ for an arbitrary convex figure $K \subset \mathbb{R}^{2}$ and an arbitrary interior point $O$ in $K$.

In [29], it was proved that the inequalities $p_{+}(K, O) \geq 6$ and $p_{-}(K, O) \geq 6$ are always valid. In [30], it was proved that for every convex figure $K \subset \mathbb{R}^{2}$ we can choose an origin $O \in \mathbb{R}^{2}$ so that $p_{+}(K, O) \leq 9$ and $p_{-}(K, O) \leq 9$. In [30], it was also proved that every f.c.f. in the bundle $\gamma_{2}^{3}$ contains a figure $K$ such that the origin $O$ of its fiber is an interior point of $K$ and $p_{-}(K, O) \leq 9$ and $p_{+}(K, O) \leq 9$.

Theorem 8. Every f.c.f. in the bundle $\gamma_{2}^{3}$ contains a figure $K$ such that $p_{+}(K, O) \leq 8$ and $p_{-}(K, O) \leq 8$ for some choice of an origin $O$ in its fiber.

Proof. We prove that a figure $K$ circumscribed about an affine-regular octagon $\Omega$ has the required property (such a figure exists by the results of [10]).

Considering support lines for $K$ at the vertices of the octagon $\Omega$, we reduce the problem to the case where $K$ is an octagon bounded by support lines. For convenience, we assume that the octagon $\Omega$ is regular and has unit width.

We prove that if we take $\Omega$ as a unit circle in the fiber under consideration, then the perimeter of the octagon $K$ circumscribed about $\Omega$ is at most 8 . Let $A_{1} A_{2}$ be a side of the octagon $K$, and let $B_{1} B_{2}$ be the chord of $\Omega$ that passes through its center $O$ and is parallel to $A_{1} A_{2}$. Let $B_{1} B_{3} B_{4} B_{2}$ be the parallelogram bounded by the continuations of two parallel sides of $K$, by the chord $B_{1} B_{2}$, and by the continuation of the segment $A_{1} A_{2}$ (see Figure 1). We have

$$
\frac{\left|A_{1} A_{2}\right|}{\left|O B_{1}\right|}=4 \frac{S\left(O A_{1} A_{2}\right)}{S\left(B_{1} B_{3} B_{4} B_{2}\right)} \leq 8 S\left(O A_{1} A_{2}\right)
$$

because, obviously, $S\left(B_{1} B_{3} B_{4} B_{2}\right) \geq 1 / 2$.

Adding the inequalities obtained for all sides of $\Omega$, we get

$$
\frac{\left|A_{1} A_{2}\right|}{\left|O B_{1}\right|}+\cdots+\frac{\left|A_{8} A_{1}\right|}{\left|O B_{8}\right|} \leq 8 S(K) \leq 8,
$$

because, obviously, $S(K) \leq 1$ (see [13]).

Remark. Apparently, the estimate obtained is unimprovable for the field of cross sections of the tetrahedron by the planes passing through its interior point. Such cross sections are triangles or quadrangles. The perimeters of the triangles are not less than 9 (see [30]). 
It is very likely that the perimeter of the quadrangle $K$ cannot be less than 8 for each choice of an interior point.

\section{§6. A PRoblem on Lipschitz FunCtions on SPHERES}

Obviously, if a function $f$ defined on the circle $S^{1}$ with angular metric satisfies the Lipschitz condition with constant $C$, then the length of the image $f\left(S^{1}\right)$ is at most $C \pi$.

Theorem 9. Let $f$ be a function defined on the spherization of the bundle $\gamma_{2}^{3}$, i.e., on the set of unit vectors in $E_{2}\left(\mathbb{R}^{3}\right)$. Assume that $f$ satisfies the Lipschitz condition with constant $C$ on each fiber (on the unit circle with angular metric). Then there is a fiber $S^{1}$ of the spherization for which the image $f\left(S^{1}\right)$ of which has length at most $C \pi / 2$.

Proof. The generalized Dyson theorem [19,3] says that, for every continuous function $f$ on the spherization of the bundle $\gamma_{2}^{3}$, there exists a fiber $S^{1} \subset E_{2}\left(\mathbb{R}^{3}\right)$ such that the function $f$ takes equal values at the vertices of some square $A B C D$ inscribed in $S^{1}$. Then, obviously, $f(A)-m \leq C \pi / 4$ and $M-f(A) \leq C \pi / 4$, where $m$ and $M$ are, respectively, the minimal and the maximal value of $f$ on the fiber (on the circle $S^{1}$ ). Consequently, $M-m \leq C \pi / 2$.

The author does not know any sharp estimate in Theorem 9.

Since there is a canonical projection $E_{k}\left(\mathbb{R}^{n}\right) \rightarrow S^{n-1}$ that takes a unit vector $\bar{e} \in$ $E_{k}\left(\mathbb{R}^{n}\right)$ to the same vector viewed as a unit vector in $\mathbb{R}^{n}$, and therefore, preserves the angles between vectors, we see that every function $f$ on the sphere $S^{n-1}$ that is $C$ Lipschitz with respect to the angular metric induces a function on the space $E_{k}\left(\mathbb{R}^{n}\right)$ that is $C$-Lipschitz on the fibers. Therefore, Theorem 9 implies the following statement.

Corollary. For every function $f$ on the sphere $S^{2} \subset \mathbb{R}^{3}$ that is $C$-Lipschitz with respect to the angular metric, there is a great circle of $S^{2}$ for which the image has length at most $C \pi / 2$.

Remarks. 1. The results of [8] imply that for any fiberwise $C$-Lipschitz continuous function $f$ on the spherization of the bundle $\gamma_{2}^{n}$, there is a fiber $S^{1} \subset E_{2}\left(\mathbb{R}^{n}\right)$ for which the image $f\left(S^{1}\right)$ has length less than $2 C \pi / p$, where $p$ is the greatest prime less than $2 n-2$.

2. The author does not know whether for all $a>0$ and $C>0$ and a positive integer $k \geq 3$ there exists $N$ with the following property: for any $n \geq N$ and an arbitrary continuous function $f$ on the spherization of the bundle $\gamma_{k}^{n}$ (i.e., on the set of unit vectors in $E_{k}\left(\mathbb{R}^{n}\right)$ ) satisfying the Lipschitz condition with constant $C$ on the fibers with angular metric, there is a fiber $S^{k-1}$ for which the image $f\left(S^{k-1}\right)$ has length not exceeding $a$.

\section{$\S 7$. Universal COVERS AND THE Young THEOREM}

A subset $A$ of $\mathbb{R}^{n}$ is called a universal cover for sets of unit diameter if every subset of $\mathbb{R}^{n}$ of unit diameter lies in a congruent copy of $A$. In [20], Pal proved that a regular hexagon of unit width is a planar universal cover. In [22], Gale proved that a regular octahedron of unit width is a universal cover in $\mathbb{R}^{3}$.

As usual, we denote by $V_{k}\left(\mathbb{R}^{n}\right)$ the Stieffel manifold of orthonormal $k$-frames in $\mathbb{R}^{n}$; let $S^{m-1} \subset \mathbb{R}^{m}$ be the unit sphere. For $v=\left(\bar{e}_{1}, \ldots, \bar{e}_{k}\right) \in V_{k}\left(\mathbb{R}^{n}\right)$, we put $-v=\left(-\bar{e}_{1}, \ldots,-\bar{e}_{k}\right) \in \mathbb{R}^{n}$. Following [21], we denote by $\mathcal{M}_{n}(N)$ the class of bounded polyhedra in $\mathbb{R}^{n}$ that are bounded by $N$ pairs of parallel hyperplanes tangent to the ball of unit diameter in $\mathbb{R}^{n}$.

Lemma 1. If there is no continuous mapping

$$
f: V_{k}\left(\mathbb{R}^{n}\right) \rightarrow S^{m-1} \subset \mathbb{R}^{m}
$$


such that $f(-v)=-f(v)$, then, for every polyhedron $\Pi \in \mathcal{M}_{k}(k+m)$, any f.c.b. of diameter at most 1 in the bundle $\gamma_{k}^{n}$ contains a body lying in a polyhedron congruent to $\Pi$.

Proof. We denote by $\ell_{1}, \ldots, \ell_{k+m}$ the axes with common origin at the center of $\Pi \in$ $\mathcal{M}_{k}(k+m)$ that are perpendicular to the pairs of parallel faces of $\Pi$. Since the polyhedron $\Pi$ is bounded, we may assume, e.g., that the axes $\ell_{1}, \ldots, \ell_{k}$ do not lie in one hyperplane in $\mathbb{R}^{k}$. Let $\bar{e}_{1}, \ldots, \bar{e}_{k+m}$ denote the unit direction vectors of the axes $\ell_{1}, \ldots, \ell_{k+m}$. Obviously, the manifold of positions of the set of vectors $\left(\bar{e}_{1}, \ldots, \bar{e}_{k+m}\right.$ ) (or of the axes $\left.\left(\ell_{1}, \ldots, \ell_{k+m}\right)\right)$ is homeomorphic to $V_{k}\left(\mathbb{R}^{n}\right)$; we denote this manifold in the same way.

For every position of the set of axes $\left(\ell_{1}, \ldots, \ell_{k+m}\right) \in V_{k}\left(\mathbb{R}^{n}\right)$, we consider the set $\left(P_{1}, \ldots, P_{k+m}\right)$ of $(k-1)$-planes in the $k$-plane of lines $\left(\ell_{1}, \ldots, \ell_{k+m}\right)$ such that the plane $P_{i}$ is perpendicular to the line $\ell_{i}$ and is equidistant from the parallel support planes for the body in the f.c.b. in question lying in the plane of the lines $\left(\ell_{1}, \ldots, \ell_{k+m}\right)$. We denote by $O$ the intersection point of $P_{1}, \ldots, P_{k}$ and place the common origin of the axes $\ell_{1}, \ldots, \ell_{k+m}$ at $O$. We define a mapping $g: V_{k}\left(\mathbb{R}^{n}\right) \rightarrow \mathbb{R}^{m}$ by taking, for $1 \leq i \leq m$, the intersection point of $\ell_{k+i} \cap P_{k+i}$ (this is a number on the number line $\ell_{k+i}$ ) for the role of the $i$ th coordinate of $g\left(\ell_{1}, \ldots, \ell_{k+m}\right)$.

By construction, $g$ is continuous and

$$
g\left(-\left(\ell_{1}, \ldots, \ell_{k+m}\right)\right)=-g\left(\ell_{1}, \ldots, \ell_{k+m}\right) .
$$

Orthogonalizing the vectors $\left(\bar{e}_{1}, \ldots, \bar{e}_{k}\right)$, we obtain a frame $v$ in $V_{k}\left(\mathbb{R}^{n}\right)$, and we put $h(v)=g\left(\ell_{1}, \ldots, \ell_{k+m}\right)$. Obviously, $h(-v)=-h(v)$. If the congruent images of the polyhedron $\Pi$ do not cover the bodies belonging to the f.c.b. in question, then for every $v \in V_{k}\left(\mathbb{R}^{n}\right)$ we have $h(v) \neq(0, \ldots, 0) \in \mathbb{R}^{m}$. Putting $f(v)=h(v) /|h(v)|$, we obtain a continuous mapping

$$
f: V_{k}\left(\mathbb{R}^{n}\right) \rightarrow S^{m-1}
$$

with the property $f(-v)=-f(v)$. Lemma 1 is proved.

Since the Stieffel manifold $V_{k}\left(\mathbb{R}^{n}\right)$ is $(n-k-1)$-connected, we see that there is no continuous mapping $f: V_{k}\left(\mathbb{R}^{n}\right) \rightarrow S^{n-k-1}$ with the property $f(-v)=-f(v)$ (see, e.g., $[15])$. We obtain the following result.

Theorem 10. For every polyhedron $\Pi \in \mathcal{M}_{k}(n)$, any f.c.b. of diameter not exceeding 1 in the bundle $\gamma_{k}^{n}$ contains a body that lies in a polyhedron congruent to $\Pi$.

Remark. It seems very likely that $\mathcal{M}_{k}(n+k-1)$ can be taken instead of $\mathcal{M}_{k}(n)$ in Theorem 10. For $k=2$ and $n=3$, this follows from the results of [21], and for even $n$ this follows from [15]. Thus, the following statement is true.

Theorem 11. If $n$ is even or $n=3$, then every f.c.f. of diameter at most 1 in the bundle $\gamma_{2}^{n}$ contains a figure lying in a regular $(2 n+2)$-gon of unit width. For all odd $n \geq 5$, every f.c.f. of diameter not exceeding 1 in the bundle $\gamma_{2}^{n}$ contains a figure lying in a regular $2 n$-gon of unit width.

For $n=2$, Theorem 11 coincides with the Pal theorem [20].

The well-known Young theorem states that any subset of $\mathbb{R}^{n}$ with diameter not exceeding 1 lies in a ball of diameter $\sqrt{2 n /(n+1)}$.

In [1], it was proved that for every $\varepsilon>0$ and every positive integer $k$, there is $N$ such that for $n \geq N$ every f.c.b. of diameter at most 1 in the bundle $\gamma_{k}^{n}$ contains a body lying in a ball of diameter not exceeding $1+\varepsilon$. This statement can easily be obtained from Theorem 10.

Theorem 11 implies the following. 
Corollary. If $n$ is even or $n=3$, then every f.c.f. of diameter at most 1 in the bundle $\gamma_{2}^{n}$ contains a figure lying in a circle of diameter sec $\frac{\pi}{2 n+2}$. For all odd $n \geq 5$, every f.c.f. of diameter at most 1 in the bundle $\gamma_{2}^{n}$ contains a figure lying in a circle of diameter $\sec \frac{\pi}{2 n}$.

The author does not know any sharp estimate for the diameter in the case where $n \geq 3$.

\section{§8. Fields OF POLYGONS AND POLYHEDRA IN UNIVERSAL BUNDLES}

A field of convex bodies in a $k$-dimensional real bundle $\gamma: E \rightarrow B$ such that all the bodies are $k$-dimensional polyhedra is called a field of polyhedra (f.p.) in the bundle $\gamma$.

These objects were considered by the author in the paper [1], where a geometric invariant $\ell(\gamma)$ of the bundle $\gamma$, namely, the smallest number $\ell$ such that $\gamma$ has an f.p. with at most $\ell$ vertices was studied.

Obviously, we have $\ell(\gamma) \leq \ell\left(\gamma_{1}\right)$ if there is a morphism of bundles $\gamma \rightarrow \gamma_{1}$ that is a fiberwise isomorphism, because we can lift the f.p. from $\gamma_{1}$ to $\gamma$ by this morphism.

In particular, $\ell(\gamma) \leq n+1$ if there exists a Gauss map $E \rightarrow \mathbb{R}^{n}$, i.e., a continuous map that is a monomorphism on the fibers. The latter condition is equivalent to the existence of a morphism that is a fiberwise isomorphism $\gamma_{k}^{n}: E_{k}\left(\mathbb{R}^{n}\right) \rightarrow G_{k}\left(\mathbb{R}^{n}\right)$ of the bundle $\gamma$ to the tautological bundle. There is an f.p. in $\gamma_{k}^{n}$ with $n+1$ vertices. This f.p. is obtained by projecting an $n$-dimensional simplex to the fibers of $\gamma_{k}^{n}$.

In [1], the following fact was conjectured.

Conjecture 1. $\ell\left(\gamma_{k}^{n}\right)=n+1$ for $k \geq 2$, i.e., every f.p. in $\gamma_{k}^{n}$ contains polyhedra with at least $n+1$ vertices.

Below, we prove that $\ell\left(\gamma_{k}^{n}\right) \geq n-k+2$.

The following simple result is in favor of Conjecture 1.

Theorem 12. Let $s_{1}, \ldots, s_{m}$ be a set of continuous sections in $\gamma_{k}^{n}$. For the role of a polyhedron in the fiber over a k-plane belonging to $G_{k}\left(\mathbb{R}^{n}\right)$ we choose the convex hull $\operatorname{conv}\left\{s_{1}(g), \ldots, s_{m}(g)\right\}$. If the resulting continuous field of figures consists of $k$ dimensional polyhedra, then $m \geq n+1$.

Proof. Indeed, in [24]-[26] it was proved that for some $g \in G_{k}\left(\mathbb{R}^{n}\right)$ we have

$$
s_{1}(g)=s_{2}(g)=\cdots=s_{n-k+1}(g),
$$

whence $m \geq n+1$.

Remark. By dimension considerations, for $n+1$ sections $s_{1}, \ldots, s_{n+1}$ of $\gamma_{k}^{n}$ in general position, the mapping

$$
g \mapsto \operatorname{conv}\left\{s_{1}(g), \ldots, s_{n+1}(g)\right\}, \quad g \in G_{k}\left(\mathbb{R}^{n}\right),
$$

gives rise to an f.p. in $\gamma_{k}^{n}$ with at most $n+1$ vertices.

The case of a continuous field of centrally-symmetric polyhedra (f.c.-s.p.) is also of interest. The following statement seems natural.

Conjecture 2. Every f.c.-s.p. in the bundle $\gamma_{k}^{n}$ contains polyhedra with $c \geq 2 n$ vertices. Observe that the f.c.-s.p. obtained by projecting an octahedron to the fibers of the bundle $\gamma_{k}^{n}$ consists of polyhedra with at most $2 n$ vertices. 
Below, we consider the treatable case of the bundle $\gamma_{2}^{n}: E_{2}\left(\mathbb{R}^{n}\right) \rightarrow G_{2}\left(\mathbb{R}^{n}\right)$. In this case, we prove Conjecture 2 and also a statement close to Conjecture 1 . Of some interest also is the invariant $\widetilde{\ell}(\gamma)$ of a vector bundle $\gamma$, equal to the smallest number $\ell$ such that $\gamma$ admits an f.p. for which the numbers of the vertices of polyhedra in different fibers differ by at most $\ell$.

Clearly, $\ell(\gamma) \geq \tilde{\ell}(\gamma)+k+1$. However, below we show that equality occurs always.

Considering the field of projections of an $n$-dimensional simplex to $k$-planes, we see that $\widetilde{\ell}\left(\gamma_{k}^{n}\right) \leq n-k$. However, we have, e.g., $\widetilde{\ell}\left(\gamma_{3}^{4}\right)=\widetilde{\ell}\left(\gamma_{7}^{8}\right)=0$, because there are fields of octahedra in $\gamma_{3}^{4}$ and $\gamma_{7}^{8}$ (see [1]).

Theorem 13. The bundles $\gamma_{3}^{4}$ and $\gamma_{7}^{8}$ admit no fields of tetrahedra.

Proof. Suppose the contrary. Let the bundle $\gamma_{3}^{4}: E_{3}\left(\mathbb{R}^{4}\right) \rightarrow G_{3}\left(\mathbb{R}^{4}\right)$ have a field of tetrahedra. Translating these tetrahedra, we may assume that their centers coincide with the origins of the fibers. The vertices of all tetrahedra in the field form a threedimensional compact submanifold $M \subset E_{3}\left(\mathbb{R}^{4}\right)$, which is a 4 -fold covering of $G_{3}\left(\mathbb{R}^{4}\right)$. Our field of tetrahedra induces a field of tetrahedra in the tautological bundle

$$
\left(\gamma_{3}^{4}\right)^{+}: E_{3}^{+}\left(\mathbb{R}^{4}\right) \rightarrow G_{3}^{+}\left(\mathbb{R}^{4}\right)
$$

over the Grassmannian manifold of oriented planes. The vertices of the tetrahedra in this field form a three-dimensional compact submanifold $\widetilde{M} \subset E_{3}^{+}\left(\mathbb{R}^{4}\right)$, which covers the base $G_{3}^{+}\left(\mathbb{R}^{4}\right) \cong S^{3}$ in a 4 -fold way. Since this base is simply connected, $\widetilde{M}$ is a disjoint union of four spheres:

$$
\widetilde{M}=S_{1} \cup S_{2} \cup S_{3} \cup S_{4} .
$$

By construction, the manifold $\widetilde{M}$ is a two-fold covering of $M$. Two distinct points in the same sphere $S_{i}$ have distinct images under the covering $\widetilde{M} \rightarrow M$, because otherwise we would have a nonzero section of the bundle $\gamma_{3}^{4}$. If points in different spheres have the same image, then we obtain a continuous field consisting of an edge of a tetrahedron in the given field. In this case, the midpoint of this edge gives a nonzero section of the bundle $\gamma_{3}^{4}$. The case of the bundle $\gamma_{7}^{8}$ is similar.

Thus, $\tilde{\ell}\left(\gamma_{3}^{4}\right)=\widetilde{\ell}\left(\gamma_{7}^{8}\right)=0$, but $\ell\left(\gamma_{3}^{4}\right)=5$ and $\ell\left(\gamma_{7}^{8}\right)=9$, and the inequality above is strict. In [1], it was proved that $\ell\left(\gamma_{n-1}^{n}\right)=n+1$ for $n \neq 4$ and $n \neq 8$, and that $\tilde{\ell}\left(\gamma_{n-1}^{n}\right)=1$ in these cases.

The author does not know the quantity $\widetilde{\ell}\left(\gamma_{k}^{n}\right)$ in the remaining cases; nevertheless, it is likely that $\tilde{\ell}\left(\gamma_{k}^{n}\right)=n-k$.

\section{§. Fields OF POLYGONS IN THE BUNDLE $\gamma_{2}^{n}$}

Theorem 14. 1. Every f.c.-s.p. in $\gamma_{2}^{n}$ contains a polygon with at least $2 n$ vertices.

2. Every f.p. in $\gamma_{2}^{n}$ contains a polygon with at least $n$ vertices.

3. If $2 n+1$ or $n+1$ is prime, then every f.p. in $\gamma_{2}^{n}$ contains a polygon with at least $n+1$ vertices.

In the sequel, we assume that $n \geq 4$ (the case where $n=3$ was considered in [1]).

We need the following Lemma 2.

For a plane figure $K \subset \mathbb{R}^{2}$, we denote by $\varepsilon(K)$ the smallest $\varepsilon>0$ such that the figure $K$ contains a circle and lies in a $(1+\varepsilon)$-homothetic circle with the same center.

Let $\mathbb{R}^{n} \subset \mathbb{R}^{n+1}$ be given by the equation $x_{n+1}=0$, and let $\ell$ be the orthogonal complement to $\mathbb{R}^{n}$, given by $x_{1}=x_{2}=\cdots=x_{n}=0$. Let $\pi: \mathbb{R}^{n+1} \rightarrow \mathbb{R}^{n}$ be the orthogonal projection. 
Lemma 2. For every $C>0$ and every f.c.f. in $\gamma_{2}^{n}$, there exists $\varepsilon>0$ such that this f.c.f. extends to an f.c.f. in $\gamma_{2}^{n+1}$ with the following properties:

1) for any plane $g \in G_{2}\left(\mathbb{R}^{n+1}\right)$ forming an angle not less than $\varepsilon$ with the line $\ell$, the orthogonal projection $\pi(K(g))$ of a figure $K(g) \subset g$ in the field in question is the figure of the same field in the plane $\pi(g) \in G_{2}\left(\mathbb{R}^{n}\right)$;

2) for any plane $g \in G_{2}\left(\mathbb{R}^{n+1}\right)$ forming an angle smaller than $\varepsilon$ with $\ell$, and any figure $K(g) \subset g$ in the field in question, we have $\varepsilon(K(g)) \geq C$.

Proof. For every $\varepsilon>0$, the first condition in the above lemma uniquely determines an extension of an f.c.f. from $\gamma_{2}^{n}$ to all planes in $G_{2}\left(\mathbb{R}^{n}\right)$ that form an angle of at least $\varepsilon$ with $\ell$. For the role of the figure $K(g)$ in such a plane $g \in G_{2}\left(\mathbb{R}^{n+1}\right)$ we must take $\pi^{-1}(K)$, where $K$ is the figure in $\pi(g) \in G_{2}\left(\mathbb{R}^{n}\right)$ that belongs to this field.

If $\varepsilon>0$ is sufficiently small and $g \in G_{2}\left(\mathbb{R}^{n+1}\right)$ is an arbitrary plane forming an angle of $\varepsilon$ with the line $\ell$, then the figure in this field that lies in $g$ has width in the direction of $\ell$ at least $C$ times greater than its width in any direction perpendicular to $\ell$.

It remains to extend the f.c.f. to the planes $g \in G_{2}\left(\mathbb{R}^{n+1}\right)$ forming angles smaller than $\varepsilon$ with $\ell$. While extending, we must preserve the property mentioned above. For instance, this can be done by ensuring that the figures in the planes passing through $\ell$ be congruent rectangles with the property that $\ell$ passes through the greatest median of each rectangle, which is at least $C$ times greater than the smallest side of the rectangle. Lemma 2 is proved.

Proof of Theorem 14. 1. We prove the first statement of the theorem.

For each plane $g \in G_{2}\left(\mathbb{R}^{n}\right)$, let $K(g)$ denote the figure of the field that lies in $g$, and let $O(g)$ be the center of symmetry of $K(g)$. We consider all ordered sets $\left\{e_{1}, \ldots, e_{p}\right\}$ of $p$ rays lying in $g$ and having origin at $O(g)$; we assume that the neighboring rays form angles of $2 \pi / p$. Obviously, the manifold $\mathcal{M}$ of the set in question is homeomorphic to the Stieffel manifold $V_{2}\left(\mathbb{R}^{n}\right)$.

Let $p$ be the smallest prime greater than $2 n-2$.

We consider the continuous mapping $F: \mathcal{M} \rightarrow \mathbb{R}^{p}$ for which the $i$ th coordinate function for $1 \leq i \leq p$ is equal to the length of the segment cut by the figure $K g$ from the ray $\ell_{i}$. The group $\mathbb{Z}_{p}$ acts on $\mathcal{M}$ by cyclic permutations of rays and on $\mathbb{R}^{p}$ by cyclic permutations of coordinates. By construction, the mapping $F$ is $\mathbb{Z}_{p}$-equivariant.

As was proved in [27], if $p+1 / 2 \leq 2 n-2$, i.e., $p \leq 4 n-5$, then the image $F(\mathcal{M})$ of $\mathcal{M}$ contains a point with $2 n-2$ equal coordinates. In other words, in the $\operatorname{set}\left(\ell_{2}, \ldots, \ell_{p}\right) \in \mathcal{M}$ we can find $2 n-2$ rays $\ell_{i_{1}}, \ldots \ell_{i_{2 n-2}}$ having the same origin at the center of symmetry of some figure $K$ in the field and such that the figure $K$ cuts out equal segments from these rays. Consequently, from the continuations of the rays $\ell_{i_{1}}, \ldots, \ell_{i_{2 n-2}}$, the figure $K$ also cuts out equal segments, and a centrally-symmetric $(4 n-4)$-gon $P$ the vertices of which coincide with those of a regular $2 p$-gon with $p \leq 4 n-5$, is inscribed in $K$. The classical results of Chebyshev imply the existence of a prime $p$ such that $2 n-2<p \leq 4 n-7$; we use this fact.

There exists a constant $C(n)$ depending on $n$ such that every polygon $P$ as described above contains a circle and is contained in a $C(n)$-homothetic circle with the same center. Indeed, assuming that the radius of the circle circumscribed about $P$ is 1 , we observe that $P$ contains a circle of radius at least $\sqrt{2} / 2$, because every side of $P$ subtends an arc of size not exceeding $90^{\circ}$, which follows from the fact that the number of the vertices of the $2 p$-gon is less than $2 \cdot(2 n-6)=4 n-12$. The convex figure $K$ that belongs to the given field and is circumscribed about $P$ lies in the star bounded by the continuations of every other side of the polygon $P$ (see Figure 2). The diameters of these stars for all such polygons $P$ are uniformly bounded because there are only finitely many forms of such polygons $P$. This proves the existence of $C(n)$. 


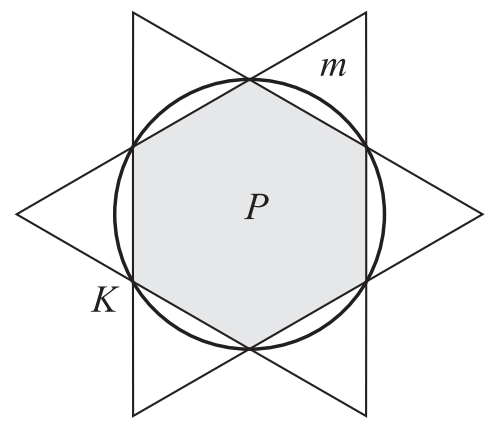

FIGURE 2

Now we complete the proof of part 1 of the theorem. Let $C(n+1)$ be the constant in $\mathbb{R}^{n+1}$ defined above for a prime $p$ such that $2 n<p<4 n-3$.

By Lemma 2, we can extend the f.c.-s.c.f. in $\gamma_{2}^{n}$ to an f.c.-s.f. in $\gamma_{2}^{n+1}$ with a constant $C>C(n+1)$. Then, by the choice of $C$, the above-mentioned centrally-symmetric $4 n$ gon $P$ inscribed in a circle can only be inscribed in a figure $K$ in $g \in G_{2}\left(\mathbb{R}^{n+1}\right)$ that forms an angle of at least $\varepsilon$ with the line $\ell=\left(\mathbb{R}^{n}\right)^{\perp}$. In this case the affine image $\pi(P)$ is inscribed in the figure $\pi(K)$ in the plane $\pi(g) \in G_{2}\left(\mathbb{R}^{n}\right)$. If the figure $\pi(K)$ is a polygon, then it has at least $2 n$ sides, because the $4 n$-gon $\pi(P)$ is inscribed in it.

2. Part 2 is proved similarly. As above, with the help of a theorem of Cohen and Lusk [27], we prove that some figure in the f.c.f. in $\gamma_{2}^{n}$ admits an inscribed affine image of a $2 n$-gon $P$ the vertices of which coincide with some vertices of a regular $p$-gon for a prime $p$ such that $2 n<p \leq 4 n-5$. Elementary considerations show that the above inequality makes it possible to estimate the "asphericity" $\varepsilon(K)$ of a convex figure $K$ circumscribed about $P$.

3. The proof of part 3 of the theorem follows from the results of the paper [9], where it was proved that if $2 n+1$ is prime, then each f.c.f. in $\gamma_{2}^{n}$ contains a figure $K$ circumscribed about an affine-regular $(2 n+1)$-gon, and if $n+1$ is prime, then it contains a figure $K$ circumscribed about an affine image of a semiregular equilateral $(2 n+2)$-gon, i.e., about a polygon with equal sides and with two interlacing series of equal angles. In all cases, if $K$ is a polygon, then we see immediately that $K$ has at least $n+1$ sides.

The next statement follows from Theorem 14 .

Corollary. Every field of polyhedra in the bundle $\gamma_{k}^{n}: E_{k}\left(\mathbb{R}^{n}\right) \rightarrow G_{k}\left(\mathbb{R}^{n}\right)$ contains a polyhedron with at least $n-k+2$ faces (vertices).

Proof. Translating the polyhedra in the field, we can reduce the proof to the case where $O$ is an interior point of all polyhedra (e.g., their barycenter). Let

$$
\mathbb{R}^{n}=L \oplus L^{\perp}
$$

be the decomposition into a direct sum of orthogonal subspaces, where $\operatorname{dim} L=k-2$. Consider a continuous field of polygons in the bundle $\gamma_{2}^{n-k+2}: E_{2}\left(L^{\perp}\right) \rightarrow G_{2}\left(L^{\perp}\right)$ :

$$
L_{1} \mapsto L_{1} \cap P,
$$

where $P$ is the $k$-dimensional polyhedron that belongs to the given field and lies in the $k$-dimensional plane $L \oplus L_{1}$. By Theorem 14, the field constructed contains a polygon with at least $n-k+2$ sides, and therefore, the given field contains a polyhedron with at least $n-k+2$ faces. 

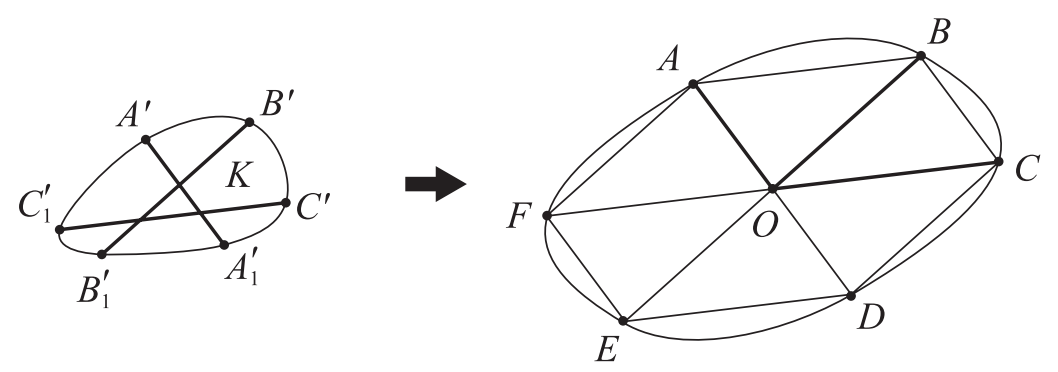

FiguRE 3

The dual bound on the number of vertices can be obtained by application of the above statement to the field of polyhedra polar to the given ones with respect to the unit balls with centers at $O$ in the fibers of $\gamma_{k}^{n}$.

\section{§10. Lattice PACKings OF CONVEX BOdies ON THE PLANE AND IN SPACE}

Fáry [4] proved that every convex figure $K \subset \mathbb{R}^{2}$ admits a lattice packing of density at least $2 / 3$. For a triangle $K$, this estimate is sharp.

Theorem 15. Every continuous field of convex figures in $\gamma_{2}^{3}$ contains a figure that admits a lattice packing in the plane with density at least $3 / 4$.

Proof. It suffices to consider the case of strictly convex figures, which is assumed in the sequel. In the remaining cases, the theorem is obtained by passing to the limit.

The proof is based on the proof of the above-mentioned Fáry theorem given in [5].

In every oriented plane $g$ passing through $O \in \mathbb{R}^{3}$, we consider the difference set

$$
\Delta K(g)=\{\bar{x}-\bar{y} \mid \bar{x}, \bar{y} \in K(g)\}
$$

for the figure $K(g)$ that belongs to the given field and lies in $g$. Our assumptions imply that $\Delta K(g)$ is a strictly convex centrally-symmetric figure. Therefore, every boundary point of $\triangle K(g)$ is a vertex $A$ of a unique affine-regular hexagon $A B C D E F$ inscribed in $\Delta K(g)$, and we may assume that the vertices in the given order lie on the boundary of $\Delta K(g)$ clockwise in accordance with the orientation of the figure. It is obvious that the manifold $\mathcal{M}$ of such hexagons is homeomorphic to $S O(3)$, and the group $\mathbb{Z}_{2}$ acts on $\mathcal{M}$ by taking a hexagon of the type $A B C D E F$ to the hexagon centrally symmetric to it.

Let $A B C D E F \in \mathcal{M}$ be an arbitrary hexagon, let $g$ be the plane where it lies, and let $O$ be the center of symmetry of $A B C D E F$ (and also the origin in $g$ ). Then the images of $K(g)$ under translations by integral linear combinations $k \overline{O A}+\ell \overline{O B}$ form a planar lattice packing of $K(\mathrm{~g})$, with the fundamental region of area $2 S(O A B)$ (see [2]). Thus, it suffices to prove that there is a plane $g$ such that $S(K(g)) \geq \frac{3}{2} S(O A B)$.

By construction, the figure $K(g)$ has chords $A_{1}^{\prime} A^{\prime}, B_{1}^{\prime} B^{\prime}$, and $C_{1}^{\prime} C^{\prime}$ that have one and the same length and are parallel to and codirected with the oriented segments $O A, O B$, and $O C$, respectively (see Figure 3 ).

We consider the continuous mapping

$$
F: \mathcal{M} \rightarrow \mathbb{R}^{2}, \quad A B C D E F \mapsto\left(\left|A^{\prime} B^{\prime}\right|-\left|A_{1}^{\prime} B_{1}^{\prime}\right|,\left|A^{\prime} B_{1}^{\prime}\right|-\left|A_{1}^{\prime} B^{\prime}\right|\right) .
$$

By construction, $F$ preserves the action of $\mathbb{Z}_{2}$, which is defined on $\mathcal{M}$ as above and on $\mathbb{R}^{2}$ as the central symmetry with respect to $(0,0)$. It is easy to check that, in this case, 

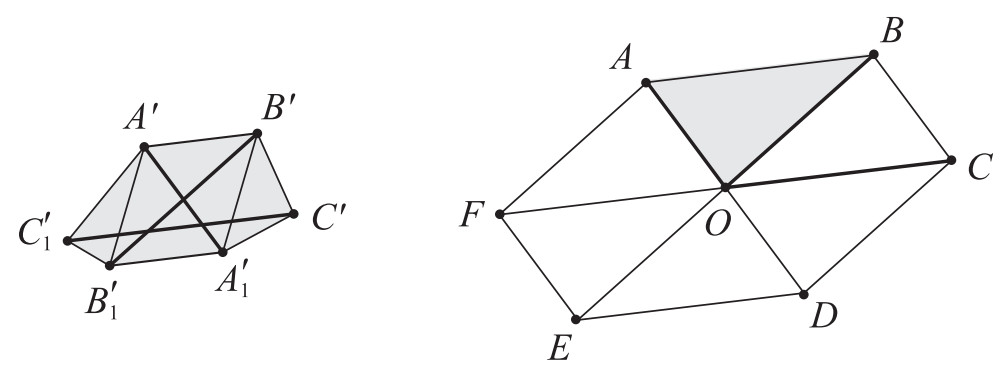

FiguRE 4

there exists a hexagon $A B C D E F$ with $F(A B C D E F)=(0,0)$ (see [21]); i.e., $A^{\prime} B^{\prime} A_{1}^{\prime} B_{1}^{\prime}$ is a parallelogram. We have

$$
\overline{A^{\prime} B^{\prime}}=\overline{B_{1}^{\prime} A_{1}^{\prime}}=\frac{1}{2} \overline{C_{1}^{\prime} C^{\prime}}, \quad S\left(A^{\prime} B^{\prime} A_{1}^{\prime} B_{1}^{\prime}\right)=S(O A B),
$$

and the area of the convex hull of the chords $A_{1}^{\prime} A^{\prime}, B_{1}^{\prime} B^{\prime}$, and $C_{1}^{\prime} C^{\prime}$ is equal to $\frac{3}{2} S(O A B)$ (see Figure 4). This completes the proof of Theorem 15.

In contrast to the Fáry theorem [4], our estimate is not sharp.

Theorem 15 implies the following statement.

Theorem 16. Every convex body $K \subset \mathbb{R}^{3}$ can be placed into a cylinder $C$ such that $V(C) \leq 3 V(K)$ and $C$ admits a lattice packing in $\mathbb{R}^{3}$ with density at least $3 / 4$.

To prove Theorem 16, we need the following lemma.

Lemma 3. Every convex body $K \subset \mathbb{R}^{n}$ lies in a cylinder $C$ of volume $V(C) \leq n V(K)$ with generatrix parallel to an arbitrary given line $\ell$.

Proof. The lines parallel to $\ell$ and passing through the points of $K \subset \mathbb{R}^{n}$ form an infinite convex cone $\widetilde{C}$ containing $K$.

Let $A B$ be the longest chord of $K$ parallel to $\ell$. Let $P_{A}$ and $P_{B}$ be support planes for the body $K$ at the points $A$ and $B$. From $\widetilde{C}$, these planes cut out a bounded cylinder $C$ that contains $K$ and has generatrix parallel to $\ell$.

After the volume-preserving Steiner symmetrization with respect to a hyperplane perpendicular to $\ell$, the cylinder $C$ becomes direct, and the body $K$ obviously contains a bipyramid mirror-symmetric with respect to the base and having the same height and base as $C$, whence $V(C) \leq n V(K)$.

Proof of Theorem 16. It suffices to observe that, by Theorem 15, the orthogonal projection $K_{1}$ of $K$ to a plane along a line $\ell$ admits a planar lattice packing with density at least $3 / 4$. By Lemma 3 , the body $K$ is inscribed in a cylinder $C$ of volume at most $3 V(K)$, with generatrix parallel to $\ell$ and with the base $K_{1}$. By construction, such a cylinder has a lattice packing of density at least $3 / 4$ in space, which proves the theorem.

Corollary. Every convex body $K \subset \mathbb{R}^{3}$ admits a lattice packing of density at least $1 / 4$.

Apparently, this estimate is very rough, because the "fibers" of the lattice packing constructed are separated from each other by parallel planes.

Using a slight modification of the proofs of Theorems 15 and 16, we can prove the following statement. 


\section{Theorem 17. Let}

$$
\gamma: G_{1}\left(\mathbb{R}^{3}\right) \times \mathbb{R}^{3} \rightarrow G_{1}\left(\mathbb{R}^{3}\right)
$$

be a trivial bundle over the Grassmannian manifold of lines passing through $O \in \mathbb{R}^{3}$. Assume that a continuous field of convex bodies is given in $\gamma$. Then the body $K$ in the fiber over some line $\ell \in G_{1}\left(\mathbb{R}^{3}\right)$ can be placed in a cylinder $C$ such that $V(C) \leq 3 V(K)$, the generatrix of $C$ is parallel to $\ell$, and $C$ has a lattice packing of density at least 3/4.

Remark. It is well known that every convex figure $K \subset \mathbb{R}^{2}$ gives rise to a lattice cover of the plane with density not exceeding $3 / 2$. The results of the paper [13] show that every f.c.f. in $\gamma_{2}^{3}$ contains a figure that yields a lattice cover of the plane with density not exceeding $\sqrt{2}$.

Acknowledgements. I want to thank the referee and the scientific editor of this paper for valuable comments, which significantly improved the original version of the paper.

\section{REFERENCES}

[1] V. V. Makeev, On some combinatorial geometry problems for vector bundles, Algebra i Analiz 14 (2002), no. 6, 169-191; English transl., St. Petersburg Math. J. 14 (2003), no. 6, 1017-1032. MR1965917 (2005b:52009)

[2] B. Grünbaum, Measures of symmetry for convex sets, Proc. Sympos. Pure Math., vol. 7, Amer. Math. Soc., Providence, RI, 1963, pp. 233-270; Borsuk's problem and related questions, ibid. pp. 271-284. MR0156259 (27:6187); MR0154183 (27:5134)

[3] V. V. Makeev, Planar sections of convex bodies and universal fibrations, Zap. Nauchn. Sem. S.Peterburg. Otdel. Mat. Inst. Steklov. (POMI) 280 (2001), 219-233; English transl., J. Math. Sci. (N.Y.) 119 (2004), no. 2, 249-256. MR1879268 (2002k:52004)

[4] I. Fáry, Sur la densité des réseaux de domaines convexes, Bull. Soc. Math. France 78 (1950), 152-161. MR0039288 (12:526a)

[5] L. Fejes Tóth, Lagerungen in der Ebene auf der Kugel und im Raum, Grundlehren Math. Wiss. in Einzeldarstellungen mit besonderer Berücksichtigung der Anwendungsgebiete, Bd. 65, SpringerVerlag, Berlin, 1953. MR0057566 (15:248b)

[6] A. Besicovitch, Measure of asymmetry of convex curves, J. London Math. Soc. 23 (1948), 237-240. $\operatorname{MR} 0027543$ (10:320r)

[7] L. G. Shnirel'man, On certain geometrical properties of closed curves, Uspekhi Mat. Nauk Vyp. 10 (1944), 34-44. (Russian) MR0012531 (7:35c)

[8] V. V. Makeev, The Knaster problem and almost spherical sections, Mat. Sb. 180 (1989), no. 3, 424-431; English transl., Math. USSR-Sb. 66 (1990), no. 2, 431-438. MR0993234 (90d:55005)

[9] _ Approximation of two-dimensional sections of convex bodies by disks and ellipses, Algebra i Analiz 16 (2004), no. 6, 162-171; English transl., St. Petersburg Math. J. 16 (2005), no. 6, 1043-1049. MR2117452 (2005i:52008)

[10] _ Affine-inscribed and affine-circumscribed polygons and polytopes, Zap. Nauchn. Sem. S.Peterburg. Otdel. Mat. Inst. Steklov. (POMI) 231 (1995), 286-298; English transl., J. Math. Sci. (N.Y.) 91 (1998), no. 6, 3518-3525. MR1434300 (98b:52004)

[11] A. Dvoretzky, Some results on convex bodies and Banach spaces, Proc. Internat. Sympos. Linear Spaces (Jerusalem, 1960), Jerusalem Acad. Press, Jerusalem, 1961, pp. 123-160. MR0139079 $(25: 2518)$

[12] V. V. Makeev, On geometric properties of three-dimensional convex bodies, Algebra i Analiz 14 (2002), no. 5, 96-109; English transl., St. Petersburg Math. J. 14 (2003), no. 5, 781-790. MR1970335 (2004c:52001)

[13] - On approximation of a three-dimensional convex body by cylinders, Algebra i Analiz 17 (2005), no. 2, 133-144; English transl., St. Petersburg Math. J. 17 (2006), no. 2, 315-323. MR2159587 (2006e:52007)

[14] R. Buck and E. Buck, Equipartition of convex sets, Math. Mag. 22 (1949), 195-198. MR0029521 $(10: 621 c)$

[15] V. V. Makeev, Some properties of continuous mappings of spheres and problems in combinatorial geometry, Geometric Questions in the Theory of Functions and Sets, Kalinin. Gos. Univ., Kalinin, 1986, pp. 75-85. (Russian) MR1027885

[16] Yu. G. Reshetnyak, An extremal problem from the theory of convex curves, Uspekhi Mat. Nauk (N.S.) 8 (1953), no. 6, 125-126. (Russian) MR0061400 (15:819d) 
[17] D. Laugwitz, Konvexe Mittelpunktsbereiche und normierte Räume, Math. Z. 61 (1954), $235-244$. MR0066667 (16:613d)

[18] V. V. Makeev, On some geometric properties of convex bodies. II, Algebra i Analiz 15 (2003), no. 6, 74-85; English transl., St. Petersburg Math. J. 15 (2004), no. 6, 867-874. MR2044632 (2004k:52021)

[19] F. J. Dyson, Continuous functions defined on spheres, Ann. of Math. (2) 54 (1951), 534-536. MR0044620 (13:450f)

[20] J. Pal, Über ein elementares Variationsproblem, Danske Vid. Selsk Mat.-Fys. Medd. 3 (1920), no. 2, $35 \mathrm{p}$.

[21] V. V. Makeev, Universal coverings. I, Ukrain. Geom. Sb. No. 24 (1981), 70-79. (Russian) MR0629813 (83e:52009)

[22] D. Gale, On inscribing n-dimensional sets in a regular n-simplex, Proc. Amer. Math. Soc. 4 (1953), 222-225. MR0053534 (14:787b)

[23] V. V. Makeev, The asphericity of the shadows of a convex body, Zap. Nauchn. Sem. S.-Peterburg. Otdel. Mat. Inst. Steklov. (POMI) 329 (2005), 67-78; English transl., J. Math. Sci. (N. Y.) 140 (2007), no. 4, 535-541. MR2215332 (2007a:52004)

[24] E. Fadell and S. Husseini, An ideal-valued cohomological index theory with applications to BorsukUlam and Bourgin-Yang theorems, Ergodic Theory Dynam. Systems 8 (1988), Charles Conley Memorial Issue, 73-85. MR0967630 (89k:55002)

[25] R. T. Živaljević and S. T. Vrećica, An extension of the ham sandwich theorem, Bull. London Math. Soc. 22 (1990), 183-186. MR1045292 (91j:52006)

[26] V. L. Dol'nikov, Transversals of families of sets in $\mathbb{R}^{n}$ and a relationship between Helly and Borsuk theorems, Mat. Sb. 184 (1993), no. 5, 111-132; English transl., Russian Acad. Sci. Sb. Math. 79 (1994), no. 1, 93-107. MR1239754 (94g:52005)

[27] F. Cohen and E. L. Lusk, Configuration-like spaces and the Borsuk-Ulam theorem, Proc. Amer. Math. Soc. 56 (1976), 313-317. MR0425949 (54:13899)

[28] A. I. Shcherba, On estimate of the perimeter of a norming figure in the Minkowski plane, 5 Internat. Conf. on Geometry and Topology in Memory A. V. Pogorelov (Cherkassy, 2003): Thesis. (Russian)

[29] _ On estimate of the perimeter of a unit disk in the Minkowski plane, Trudy Rubtsov. Industr. Inst. Vyp. 12 (2003), 96-107. (Russian)

[30] V. V. Makeev, An upper bound for the perimeter of a nonsymmetric unit disk in the Minkowski plane, Zap. Nauchn. Sem. S.-Peterburg. Otdel. Mat. Inst. Steklov. (POMI) 299 (2003), 262-266; English transl., J. Math. Sci. (N.Y.) 131 (2005), no. 1, 5406-5408. MR2038827 (2005a:52007)

Department of Mathematics and Mechanics, St. Petersburg State University, UniverSitetskiI Pr. 28, Stary ̌ Peterhof, St. Petersburg 198504, Russia

E-mail address: mvv57@inbox.ru

Received 14/FEB/2005

Translated by B. M. BEKKER 